\title{
Rest-Activity Pattern and Circadian Phase Alterations Across the Alzheimer's Disease Clinical Spectrum
}

\author{
Hyun Woong Roh and Sang Joon Son \\ Department of Psychiatry, Ajou University School of Medicine, Suwon, Korea
}

\begin{abstract}
Actigraphy-derived locomotor activity recordings are novel and critical tools for evaluating rest-activity pattern and circadian phase in humans. We conducted a narrative review assessing rest-activity pattern and circadian phase alterations within various stages of Alzheimer's disease, in consideration of the reciprocal associations between neurodegeneration and circadian rhythm disruption in patients with Alzheimer's disease. The goal of this review was to characterize possible associations between circadian rhythm disruption and neurodegeneration in Alzheimer's disease. To the best of our knowledge, only two studies have assessed rest-activity pattern and circadian phase alterations in the preclinical Alzheimer's disease stage and the results of the studies were inconsistent. Several studies have evaluated rest-activity pattern and circadian phase alterations in patients with Alzheimer's dementia. The most replicated findings were delayed phase and increased activity fragmentation, represented as increased intra-daily variability. Unfortunately, many studies performed in dementia patients have not examined neuroimaging biomarkers or structured neuropsychological tests, thus limiting the specification of dementia clinical diagnoses. Future studies should consider a more comprehensive evaluation of various clinical and biomarker characteristics in patients with dementia or Alzheimer's disease.
\end{abstract}

Keywords: Actigraphy; Alzheimer's disease; Circadian phase; Rest-activity pattern

Received: November 30, 2021 Revised: December 10, 2021 Accepted: December 11, 2021

Corresponding author: Hyun Woong Roh, MD, PhD, Department of Psychiatry, Ajou University School of Medicine, 164 World cup-ro, Yeongtong-gu, Suwon 16499, Korea.

Tel: 82-31-219-7851, Fax: 82-31-219-5179, E-mail: hansin8607@ajou.ac.kr

(a) This is an Open Access article distributed under the terms of the Creative Commons Attribution Non-Commercial License (https://creativecommons.org/licenses/bync/4.0) which permits unrestricted non-commercial use, distribution, and reproduction in any medium, provided the original work is properly cited.

\section{INTRODUCTION}

Circadian rhythms are $24 \mathrm{~h}$ oscillations within biochemical and cellular life processes [1]. The first mammalian clock gene, CLOCK, was identified in 1994 [2], and several mammalian clock genes (i.e., PER1, PER2, NR1D1, BMAL1) have been discovered since [3-5]. The mammalian cellular circadian clock is based on autonomous transcriptional and translational feedback loops comprising these clock genes. Additionally, the suprachiasmatic nucleus ( $\mathrm{SCN}$ ) of the hypothalamus is a mammalian master clock that generates and maintains physiological and behavioral oscillations, with SCN lesions affecting circadian temperature and rest-activity rhythms [6-8].

Diurnal rest-activity patterns are critical circadian rhythm outputs generated by the SCN. Several rest-activity pattern alterations have been reported with aging, including fragmented and blunted rest-activity rhythms [9]. Additionally, impacts on the advanced phase of rest-activity and sleep patterns are among the most well-known characteristics in older adults. Older adults tend to sleep earlier in the evening and to wake up earlier than desired [10]. However, controversy exists as to whether rest-activity pattern or circadian phase alterations are driven by normal physiological aging processes or by pathological neurodegenerative processes $[11,12]$. Reciprocal connections among circadian rhythm disruption and neurodegenerative processes also need to be considered. For example, recent animal studies using a genetic circadian rhythm deficit model suggest that circadian disruption in animals can accelerate neurodegenerative processes by affecting biological mechanisms associated with Alzheimer's disease [13-16]. In contrast, neurodegeneration in rest-activity and sleepregulating regions likewise presents a critical mechanism affecting rest-activity patterns or circadian phases. For example, the lo- 
cus coeruleus is one of the most critical brain regions responsible for rest-activity oscillations, sleep-wake cycles, attention, learning, and memory function [17]. Interestingly, recent experimental and clinical studies suggest that tau accumulation and subsequent neurodegeneration in the locus coeruleus may occur at a very early stage of Alzheimer's disease [17-19].

Actigraphy-derived locomotor activity recordings are novel and powerful tools for assessing rest-activity pattern and circadian phase alterations [20]. Considering the reciprocal relationships with respect to neurodegeneration and circadian rhythm disruption among patients with neurodegenerative disorders, assessing rest-activity pattern and circadian phase changes within various stages of neurodegenerative disease can be helpful in understanding the temporal and causal relationships between circadian rhythm disruption and neurodegeneration [21]. Rest-activity pattern and circadian phase alterations might serve not only as a biomarker for diagnosis but also as disease course or prognosis of Alzheimer's disease. In addition, intervention to optimize or restore circadian function can be considered as a treatment option in patients with Alzheimer's disease. The purpose of this article is to review the historical and recent evidence regarding potential associations of actigraphy-derived rest-activity pattern and circadian phase parameters with clinical or biomarker characteristics in patients with Alzheimer's disease.

\section{ACTIGRAPHY-DERIVED REST-ACTIVITY PATTERN AND CIRCADIAN PHASE: NONPARAMETRIC AND COSINOR ANALYSES}

Nonparametric analysis does not present a priori assumptions for the waveform of diurnal activity rhythm. Instead, this modality evaluates rest-activity and circadian phase based on raw activity counts [22]. Parameters for nonparametric analysis include 1) interdaily stability (IS, representing the strength of coupling with respect to rhythms and environmental zeitgebers), 2) intradaily variability (IV, representing rest-activity pattern fragmentation in one day), 3) relative amplitude (representing rest-activity rhythm amplitude), 4) the least active $5 \mathrm{~h}$ (L5) onset time, and 5) the most active $10 \mathrm{~h}$ (M10) onset time.

In contrast, cosinor analysis fits activity counts to cosine curves using least-square methods for assessing rest-activity and circadian phase [23]. Parameters for cosinor analysis include 1) robustness (i.e., goodness of fit for the cosine curve, representing the strength of rhythms within circadian activity),2) midline estimation of rhythm statistics (representing the mean of the activity fitted curve), 3) amplitude (peak to nadir differences in activity, representing circadian activity amplitude), and 4 ) acrophase (timing of peak activity, representing the rest-activity phase).

\section{REST-ACTIVITY PATTERN AND CIRCADIAN PHASE ALTERATIONS IN PRECLINICAL ALZHEIMER'S DISEASE OR MILD COGNITIVE IMPAIRMENT}

In the study profiled here, participants with preclinical Alzheimer's disease were identified as cognitively normal individuals with Alzheimer's-associated brain biomarkers, such as amyloid deposition in the brain identified via positron emission tomography (PET), as well as Alzheimer's-associated tau and amyloid protein levels measured in the cerebrospinal fluid [24]. These findings suggest that Alzheimer's disease starts long before various symptoms become apparent. Although participants at the preclinical Alzheimer's disease stage are usually identified only in research settings, Musiek et al. [25] carefully assessed rest-activity alterations and circadian phase markers in 189 cognitively normal older adults via PET; this study enrolled 50 older adults positive for amyloid deposition in the brain. The researchers showed that increased IV, indicating rest-activity pattern fragmentation, was associated with an increased cerebrospinal fluid phosphorylated-tau to amyloid beta- 42 ratio. Additionally, the researchers found that aging was associated with several circadian rest-activity parameters independent of preclinical Alzheimer's disease pathology, especially in men. Another study performed in the Republic of Korea demonstrated that participants with preclinical Alzheimer's disease present with an earlier circadian phase upon actigraphy [26]. In this study, the researchers suggested that preclinical Alzheimer's disease made the effects of age-associated phase advance more prominent. However, the patients, who were clinically diagnosed with Alzheimer's disease, exhibited a later circadian phase on actigraphy. As of the current review, the above two results are the only evidence to date suggesting possible associations of preclinical Alzheimer's disease pathology with restactivity pattern and circadian phase in patients in the preclinical stage of the disease.

Mild cognitive impairment (MCI) due to Alzheimer's disease is a stage between preclinical Alzheimer's disease and dementia due to Alzheimer's disease [27]. Patients with MCI usually have objective cognitive impairment but do not have difficulties in activities of daily functioning [28]. Several reports have assessed rest-activity pattern and circadian phase alterations within MCI. For example, in 2014, Ortiz-Tudela et al. [29] assessed rest-activity and temperature rhythms in 21 participants with MCI via actigraphy and found that participants with MCI present with phase advances in both activity and temperature rhythms as compared with healthy controls. Another study performed in Hong Kong demonstrated different results [30]. In this study, Lee et al. [30] recruited 174 older adults (including 123 older adults with normal cognition and 51 patients with MCI). There were no statistically significant baseline differences in rest-activity pattern or circadian phase when comparing cognitively normal older adults and patients with MCI in this study. However, multinomial logistic regression suggested that participants showing delayed acro- 
phase upon cosinor analysis were more likely to present with worse cognitive function. Another study conducted in the Republic of Korea that enrolled 70 participants with MCI and 30 with mild dementia suggested that amyloid-positive participants had a later L5 onset time on nonparametric analysis as compared with their amyloid-negative counterparts [31]. However, these researchers did not evaluate rest-activity pattern or circadian phase in cognitively normal older adults or in healthy controls. Finally, in 2020, Li et al. [32] published study results regarding longitudinal associations of rest-activity patterns and circadian phase parameters with clinical conversion of normal cognition to MCI or Alzheimer's dementia. This study enrolled 1,401 older adults (aged >59 years) from within the Rush Memory and Aging Project and found that an increased risk of Alzheimer's dementia conversion among patients with MCI can be predicted via lower amplitude, higher IV, and lower IS. Additionally, the researchers also found that circadian amplitude, acrophase, and IS decreased with aging and that IV increased with aging in the older adult population. This study suggests a potential bidirectional relationship and common underlying pathophysiological mechanisms between circadian rhythm disruption and Alzheimer's disease progression.

\section{REST-ACTIVITY PATTERN AND CIRCADIAN PHASE ALTERATIONS IN DEMENTIA DUE TO ALZHEIMER'S DISEASE}

Rest-activity pattern and circadian phase alterations have also been evaluated in patients with dementia occurring due to Alzheimer's disease. One historical report was published in 1996 by van Someren et al. [33]. This study assessed 34 patients with Alzheimer's disease who were living at home or in a nursing home and showed that changes in rest-activity patterns were stronger in the institutionalized patients. Additionally, this study assessed the possible associations of rest-activity patterns and circadian phase parameters with daytime activity and environmental light exposure; daytime activity and environmental light exposure were found to be meaningful predictive factors. Another critical report was published by Hatfield et al. [34] in 2004. In this study, patients with moderate Alzheimer's disease presented with decreased IS, increased IV, and decreased amplitude upon actigraphy. However, the degree of disruption for each parameter was not specifically correlated with dementia severity.

In 2015, a study conducted by Wang et al. [35] suggested a possible association between rest-activity patterns and vasoactive intestinal peptide-expressing SCN neuron neurodegeneration among older adults. In this study, the number of vasoactive intestinal peptide immunoreactive neurons in the SCN was positively associated with circadian rhythm amplitude with respect to motor activity in both patients with Alzheimer's disease and in age-matched healthy controls. Additionally, patients with Alzheimer's disease exhibited a delayed circadian phase as compared with healthy controls. In 2016, La Morgia et al. [36] reported that 16 partici- pants with mild to moderate Alzheimer's disease presented with reduced relative amplitude and daytime activities as compared with 10 healthy control participants. In this study, the researchers stated that rest-activity patterns and their dysfunctions appear to exhibit substantial variability between individuals. Our team presents similar results with respect to insights into the large individual variability among rest-activity patterns and circadian phase parameters in this regard. For example, in a prior paper, we extracted data on rest-activity patterns and circadian phase parameters from 100 patients with MCI or mild dementia enrolled in the Biobank Innovation for Chronic Cerebrovascular Disease with Alzheimer's Disease Study and found that rest-activity patterns and circadian phase parameters were normally distributed with a large variance [31]. Additionally, recently published articles have evaluated not only sleep, rest-activity patterns, and circadian phase parameters, but have also investigated postmortem neocortical microglial marker gene expression with respect to measured cognitive function [37]. A total of 685 older adults (265 with Alzheimer's dementia and 420 without) were evaluated in the latter study, although only a subset of study participants had postmortem neocortical gene expression data. This study demonstrated that greater sleep fragmentation upon actigraphy may be associated with higher neocortical microglial marker gene expression and worse cognitive function. However, the researchers did not find a statistically significant association between circadian rhythmicity (IS) and microglial marker gene expression levels. These findings suggest that microglial activation may be specifically associated with sleep fragmentation and not with circadian rhythmicity. However, caution is needed in the interpretation of these results, as this investigation did not evaluate other rest-activity or circadian phase variables, such as IV or amplitude.

Several additional studies have evaluated potential cross-sectional or longitudinal associations of rest-activity patterns and circadian phase parameters with clinical and biomarker characteristics in patients with dementia [38-42]. However, clinical or biomarker-based Alzheimer's disease diagnoses were not performed in these investigations. Considering the potential moderating effects of cortical Alzheimer's disease pathology on restactivity pattern, and possible noise effects of resting tremors on rest-activity pattern parameters in patients with Lewy body dementia, future research should consider the specification of dementia clinical diagnoses, such as Alzheimer's dementia, vascular dementia, frontotemporal dementia, and Lewy body dementia. Recently developed neuroimaging biomarkers and detailed clinical evaluations with structured neuropsychological tests need to be considered in future research as well.

\section{CONCLUSION}

This narrative review provides a comprehensive presentation with respect to actigraphy-derived rest-activity pattern and circadian phase alterations in patients with Alzheimer's disease. To the best of our knowledge, only two previous studies have assessed 
rest-activity patterns and circadian phase in the preclinical Alzheimer's disease stage. These studies have shown inconsistent results. Additionally, several studies have assessed rest-activity patterns and circadian phase alterations among patients with Alzheimer's dementia. The most replicated findings demonstrate delayed phase and increased activity fragmentation, represented as increased IV on actigraphy readings. Unfortunately, many studies assessing rest-activity patterns in dementia have not evaluated neuroimaging biomarkers and have not conducted structured neuropsychological examinations. This has limited the specification of dementia clinical diagnoses within many investigations. In addition, actigraphy-derived rest-activity pattern and circadian phase parameters are likely to be influenced by several confounding factors such as physical activity, mealtime, weekend, and seasonal effect. The influence of confounding factors can be minimized by using clinical and research guideline such as the Society of Behavioral Sleep Medicine guideline [43]. Future studies should consider a more comprehensive assessment of a range of clinical and biomarker characteristics among patients with dementia due to Alzheimer's disease.

\section{Acknowledgments}

This work was also supported and funded by the grant from $\mathrm{Na}$ tional Research Foundation of Korea (NRF), funded by the Ministry of Science and ICT (NRF-2019R1A5A2026045) and the grant from the Korea Health Technology R\&D Project through the Korea Health Industry Development Institute (KHIDI), and the Ministry of Health \& Welfare, Republic of Korea (grant number: HR21C1003).

\section{Conflicts of Interest}

The authors have no potential conflicts of interest to disclose.

\section{Author Contributions}

Conceptualization: Hyun Woong Roh. Writing-original draft: Hyun Woong Roh. Writing_review \& editing: Sang Joon Son.

\section{ORCID iDs}

Hyun Woong Roh (D)

https://orcid.org/0000-0002-1333-358X

Sang Joon Son (D)

https://orcid.org/0000-0001-7434-7996

\section{REFERENCES}

1. Reppert SM, Weaver DR. Coordination of circadian timing in mammals. Nature 2002;418:935-941.

2. Vitaterna MH, King DP, Chang AM, Kornhauser JM, Lowrey PL, McDonald JD, et al. Mutagenesis and mapping of a mouse gene, clock, essential for circadian behavior. Science 1994;264:719-725.

3. Shearman LP, Zylka MJ, Weaver DR, Kolakowski LF Jr, Reppert SM. Two period homologs: circadian expression and photic regulation in the suprachiasmatic nuclei. Neuron 1997;19:1261-1269.

4. Ikeda M, Nomura M. cDNA cloning and tissue-specific expression of a novel basic helix-loop-helix/PAS protein (BMAL1) and identification of alternatively spliced variants with alternative translation initiation site usage. Bio- chem Biophys Res Commun 1997;233:258-264

5. Preitner N, Damiola F, Lopez-Molina L, Zakany J, Duboule D, Albrecht U, et al. The orphan nuclear receptor REV-ERBalpha controls circadian transcription within the positive limb of the mammalian circadian oscillator. Cell 2002;110:251-260.

6. Weaver DR. The suprachiasmatic nucleus: a 25-year retrospective. J Biol Rhythms 1998;13:100-112.

7. Eastman CI, Mistlberger RE, Rechtschaffen A. Suprachiasmatic nuclei lesions eliminate circadian temperature and sleep rhythms in the rat. Physiol Behav 1984;32:357-368.

8. Ibuka N, Inouye SI, Kawamura H. Analysis of sleep-wakefulness rhythms in male rats after suprachiasmatic nucleus lesions and ocular enucleation. Brain Res 1977;122:33-47.

9. Hood S, Amir S. The aging clock: circadian rhythms and later life. J Clin Invest 2017;127:437-446.

10. Li J, Vitiello MV, Gooneratne NS. Sleep in normal aging. Sleep Med Clin 2018;13:1-11.

11. Nakamura TJ, Takasu NN, Nakamura W. The suprachiasmatic nucleus: agerelated decline in biological rhythms. J Physiol Sci 2016;66:367-374.

12. Logan RW, McClung CA. Rhythms of life: circadian disruption and brain disorders across the lifespan. Nat Rev Neurosci 2019;20:49-65.

13. Kress GJ, Liao F, Dimitry J, Cedeno MR, FitzGerald GA, Holtzman DM, et al. Regulation of amyloid- $\beta$ dynamics and pathology by the circadian clock. J Exp Med 2018;215:1059-1068.

14. Musiek ES, Lim MM, Yang G, Bauer AQ, Qi L, Lee Y, et al. Circadian clock proteins regulate neuronal redox homeostasis and neurodegeneration. J Clin Invest 2013;123:5389-5400.

15. Nakazato R, Kawabe K, Yamada D, Ikeno S, Mieda M, Shimba S, et al. Disruption of Bmall impairs blood-brain barrier integrity via pericyte dysfunction. J Neurosci 2017;37:10052-10062.

16. Lananna BV, Nadarajah CJ, Izumo M, Cedeño MR, Xiong DD, Dimitry J, et al. Cell-autonomous regulation of astrocyte activation by the circadian clock protein BMAL1. Cell Rep 2018;25:1-9.e5.

17. James T, Kula B, Choi S, Khan SS, Bekar LK, Smith NA. Locus coeruleus in memory formation and Alzheimer's disease. Eur J Neurosci 2021;54:69486959.

18. Betts MJ, Kirilina E, Otaduy MCG, Ivanov D, Acosta-Cabronero J, Callaghan MF, et al. Locus coeruleus imaging as a biomarker for noradrenergic dysfunction in neurodegenerative diseases. Brain 2019;142:2558-2571.

19. Matchett BJ, Grinberg LT, Theofilas P, Murray ME. The mechanistic link between selective vulnerability of the locus coeruleus and neurodegeneration in Alzheimer's disease. Acta Neuropathol 2021;141:631-650.

20. Leng Y, Musiek ES, Hu K, Cappuccio FP, Yaffe K. Association between circadian rhythms and neurodegenerative diseases. Lancet Neurol 2019;18: 307-318.

21. Musiek ES. Circadian rhythms in AD pathogenesis: a critical appraisal. Curr Sleep Med Rep 2017;3:85-92.

22. Witting W, Kwa IH, Eikelenboom P, Mirmiran M, Swaab DF. Alterations in the circadian rest-activity rhythm in aging and Alzheimer's disease. Biol Psychiatry 1990;27:563-572.

23. Ancoli-Israel S, Cole R, Alessi C, Chambers M, Moorcroft W, Pollak CP. The role of actigraphy in the study of sleep and circadian rhythms. Sleep 2003; 26:342-392.

24. Dubois B, Hampel H, Feldman HH, Scheltens P, Aisen P, Andrieu S, et al. Preclinical Alzheimer's disease: definition, natural history, and diagnostic criteria. Alzheimers Dement 2016;12:292-323.

25. Musiek ES, Bhimasani M, Zangrilli MA, Morris JC, Holtzman DM, Ju YS. Circadian rest-activity pattern changes in aging and preclinical Alzheimer disease. JAMA Neurol 2018;75:582-590.

26. Park JE, Lee YJ, Byun MS, Yi D, Lee JH, Jeon SY, et al. Differential associations of age and Alzheimer's disease with sleep and rest-activity rhythms across the adult lifespan. Neurobiol Aging 2021;101:141-149.

27. Sperling RA, Aisen PS, Beckett LA, Bennett DA, Craft S, Fagan AM, et al. Toward defining the preclinical stages of Alzheimer's disease: recommendations from the National Institute on Aging-Alzheimer's Association workgroups on diagnostic guidelines for Alzheimer's disease. Alzheimers Dement 2011;7:280-292.

28. Winblad B, Palmer K, Kivipelto M, Jelic V, Fratiglioni L, Wahlund LO, et al. Mild cognitive impairment--beyond controversies, towards a consensus: re- 
port of the International Working Group on mild cognitive impairment. J Intern Med 2004;256:240-246.

29. Ortiz-Tudela E, Martinez-Nicolas A, Díaz-Mardomingo C, García-Herranz S, Pereda-Pérez I, Valencia A, et al. The characterization of biological rhythms in mild cognitive impairment. Biomed Res Int 2014;2014:524971.

30. Lee PMY, Ling Kwok BH, Ting Ma JY, Tse LA. A population-based prospective study on rest-activity rhythm and mild cognitive impairment among Hong Kong healthy community-dwelling older adults. Neurobiol Sleep Circadian Rhythms 2021;10:100065.

31. Roh HW, Choi JG, Kim NR, Choe YS, Choi JW, Cho SM, et al. Associations of rest-activity patterns with amyloid burden, medial temporal lobe atrophy, and cognitive impairment. EBioMedicine 2020;58:102881.

32. Li P, Gao L, Gaba A, Yu L, Cui L, Fan W, et al. Circadian disturbances in Alzheimer's disease progression: a prospective observational cohort study of community-based older adults. Lancet Healthy Longev 2020;1:e96-e105.

33. van Someren EJ, Hagebeuk EE, Lijzenga C, Scheltens P, de Rooij SE, Jonker C, et al. Circadian rest-activity rhythm disturbances in Alzheimer's disease. Biol Psychiatry 1996;40:259-270.

34. Hatfield CF, Herbert J, van Someren EJ, Hodges JR, Hastings MH. Disrupted daily activity/rest cycles in relation to daily cortisol rhythms of homedwelling patients with early Alzheimer's dementia. Brain 2004;127:10611074 .

35. Wang JL, Lim AS, Chiang WY, Hsieh WH, Lo MT, Schneider JA, et al. Suprachiasmatic neuron numbers and rest-activity circadian rhythms in older humans. Ann Neurol 2015;78:317-322.
36. La Morgia C, Ross-Cisneros FN, Koronyo Y, Hannibal J, Gallassi R, Cantalupo G, et al. Melanopsin retinal ganglion cell loss in Alzheimer disease. Ann Neurol 2016;79:90-109.

37. Kaneshwaran K, Olah M, Tasaki S, Yu L, Bradshaw EM, Schneider JA, et al. Sleep fragmentation, microglial aging, and cognitive impairment in adults with and without Alzheimer's dementia. Sci Adv 2019;5:eaax7331.

38. Ishimaru D, Tanaka H, Nagata Y, Ogawa Y, Fukuhara K, Takabatake S, et al. Impact of disturbed rest-activity rhythms on activities of daily living in moderate and severe dementia patients. Alzheimer Dis Assoc Disord 2021; 35:135-140.

39. Gehrman P, Marler M, Martin JL, Shochat T, Corey-Bloom J, Ancoli-Israel $S$. The relationship between dementia severity and rest/activity circadian rhythms. Neuropsychiatr Dis Treat 2005;1:155-163.

40. Hooghiemstra AM, Eggermont LH, Scheltens P, van der Flier WM, Scherder EJ. The rest-activity rhythm and physical activity in early-onset dementia. Alzheimer Dis Assoc Disord 2015;29:45-49.

41. Smagula SF, Gujral S, Capps CS, Krafty RT. A systematic review of evidence for a role of rest-activity rhythms in dementia. Front Psychiatry 2019;10: 778.

42. Song Y, Dowling GA, Wallhagen MI, Lee KA, Strawbridge WJ, Hubbard EM. Rest-activity patterns in institutionalized Korean older adults with dementia: a pilot study. J Gerontol Nurs 2009;35:20-28; quiz 30-31.

43. Ancoli-Israel S, Martin JL, Blackwell T, Buenaver L, Liu L, Meltzer LJ, et al. The SBSM guide to actigraphy monitoring: clinical and research applications. Behav Sleep Med 2015;13 Suppl 1:S4-S38. 